\title{
La construcción del espacio turístico \\ a través de la fraseología metafórica*
}

\author{
Sara Piccioni (Chieti-Pescara), Gianluca Pontrandolfo (Trieste)
}

\begin{abstract}
This article explores the metaphorical strategies conveyed by phraseological units in a subsection of the Linguaturismo corpus (Calvi 2011). Through a quali-quantative analysis, metaphorical phraseological units related to space were identified and classified, with a view to identifying the persuasive strategies necessary to create the image of the tourist destination in the reader/traveler's mind.

The analysis followed a three-step methodology: a) semi-automatic elaboration of a list of potentially metaphorical $\mathrm{N}$-grams; b) identification of phraseological patterns in the $\mathrm{N}$-grams according to syntactic criteria (Corpas Pastor 1996); c) classification of metaphorical phraseological units based on the conceptual domain of space used as source domain, as target domain, and as both source and target domains (Lakoff/Johnson 1980).

Results show that space metaphors in tourist texts rely on conventional linguistic patterns typical of general language. However, on a conceptual level, they display a functional specialisation as persuasive devices; by placing the readers/tourists at the centre of the communication, metaphors contribute to enhancing their sensorial perception, thus inviting them to visit a destination, buy a tourist service, or do a tourist experience.
\end{abstract}

\section{$1 \quad$ Introducción}

La idea de que el léxico del turismo se caracteriza también por la presencia de unidades fraseológicas (UUFF) no es nueva, aunque el tema no haya recibido la atención debida en la literatura del sector. Ya en 2006, Calvi subrayaba la existencia de palabras "que se sitúan en el área periférica del léxico del turismo" y que, connotando la fascinación del lugar turístico, "[dan] lugar a combinaciones recurrentes (aguas cristalinas, idílicas playas, selvas inexploradas, espléndidas visitas, panorámicos paisajes, etc.)" (2006: 59).

Con respecto al uso de las metáforas en este lenguaje sectorial, la autora señala su escasez lingüística debida a las características del objeto de estudio (Calvi 2000: 69-70). Muchas de las formaciones neológicas se acuñan combinando pocos vocablos recurrentes ('turismo', 'ruta',

\footnotetext{
* El presente artículo es fruto del trabajo conjunto de los dos autores. Por razones de reconocimiento académico, se precisa que los párrafos $\S 1, \S 2, \S 4$ y $\$ 7$ corren a cargo de Gianluca Pontrandolfo; los párrafos $\$ 3$, §5 y §6 están redactados por Sara Piccioni.
} 
etc.) con distintos sustantivos o adjetivos, mientras que los términos de otros sectores disciplinares (historia del arte, geografía, etc.) se usan en su significado originario y no sufren un proceso de redefinición semántica. Sin embargo, la estudiosa presenta algunos ejemplos clásicos como los colores -el blanco metáfora de la nieve ('semana blanca'), el verde del turismo natural, el azul de las playas ('bandera azul')- así como algunas resemantizaciones como 'paquete' con referencia a los servicios ofrecidos por las agencias en la organización de viajes.

Lo que sí resulta evidente es la función persuasiva que desempeñan indirectamente las unidades fraseológicas metafóricas en la comunicación turística. De hecho, al encender la imaginación de los turistas creando en sus mentes imágenes casi cinematográficas del lugar turístico' (Urry 2001), estas expresiones se proponen influir en la conducta del interlocutor, convenciéndole a visitar una meta o a adquirir un servicio turístico.

Si bien el binomio fraseología y metáfora se haya explorado en literatura tanto en la lengua general (véanse, entre otros, Luque Durán/Manjón, 1998; Pamies Bertrán 2002; Messina Fajardo 2017) como en las lenguas de especialidad (véanse Ramos-Ruiz 2015; Caruso 2016), todavía no ha recibido la debida atención en el marco de la comunicación turística, donde son escasos los estudios empíricos capaces de revelar cómo se emplea la fraseología metafórica y con qué tipologías y estrategias en el lenguaje del turismo. Con la excepción del estudio de Corbacho Sánchez (2014) sobre fraseología metafórica, las investigaciones llevadas a cabo en este ámbito pertenecen básicamente a dos grupos: a) las que se ciñen al aspecto metafórico (Barcelona Sánchez/Rocamora Abellán 2000; Francesconi 2008; Mattiello 2012; Cappelli 2012), sin ninguna relación explícita con la fraseología; b) las que analizan los fraseologismos y las expresiones metafóricas desde una perspectiva estrictamente cultural y traductológica (p. ej., Navarro Coy/Soto Almela 2014).

Por lo que atañe a los estudios de corte general y no sectorial, cabe señalar que la mayoría se centra en enunciados fraseológicos como refranes o enunciados de valor específico, más que en colocaciones y locuciones, siguiendo la clasificación de Corpas Pastor (1996) (véase §4). Los estudios sobre el lenguaje turístico, en cambio, se focalizan en las metáforas como medio para representar de forma icónica la ruta que los ojos del 'turista-niño' (Dann 1996) tendrían que seguir.

Pues bien, la presente investigación se propone estudiar las estrategias metafóricas utilizadas en los textos turísticos con función persuasiva a partir de la identificación de las unidades fraseológicas más frecuentes presentes en el corpus Linguaturismo (véase §3). El objetivo último es comprobar cómo la fraseología metafórica contribuye a la creación de la imagen del destino turístico. En particular, el análisis se centra en las expresiones metafóricas relacionadas con el ESPACIO, ya que estas -en línea con los resultados de otros estudios (p. ej. Cappelli 2012)tienen una relevancia cognitiva especial en los textos turísticos debido a la centralidad de la experiencia del espacio en la realidad turística.

\footnotetext{
1 "With their lexical choices, specialist-writers guide their non-specialist readers through their tourist experience so that they become part of the scene described. In choosing the most suitable verb, capable of conveying both spatial and emotional information at the same time, the writer fires the readers' imagination by creating in their mind an almost cinematic image and contributes to building the (global) tourist gaze (Urry 2001)" (Cappelli 2012: $33)$.
} 
Desde la vertiente metodológica, el estudio arranca de la consideración de que, en realidad, la proyección metafórica es una propiedad definitoria de los fraseologismos (Dobrovol'skij 1998: 57, citado en Pamies Bertrán 2002: 11); por ende, las unidades fraseológicas pueden representar un medio eficaz para detectar patrones metafóricos en el discurso turístico.

Dos hipótesis guían el estudio: por un lado, se preve que las metáforas espaciales en textos turísticos responden a patrones lingüísticos convencionales propios de la lengua general; por otro lado, a nivel conceptual, se postula una especialización funcional de estas metáforas convencionales en los textos turísticos como instrumentos de persuasión que, poniendo al centro de la comunicación al lector/viajero como sujeto que percibe, contribuyen a reforzar su percepción sensorial para invitarlo a visitar un determinado lugar, comprar un servicio turístico, etc.

\section{Fraseología, metáforas y discurso turístico}

Nadie puede cuestionar la relevancia de la fraseología en los géneros discursivos turísticos. Se trata, evidentemente, de uno de los rasgos que confiere estabilidad y reconocibilidad a estos géneros y que contribuye a crear ese 'horizonte de expectativas' en el destinatario del texto.

Según el nivel de especialización y las relaciones horizontales y verticales presentes en la interacción (véase Calvi 2009), la comunicación turística impone pautas discursivas diferenciadas y estructuras lingüísticas, terminológicas y fraseológicas diferentes. Efectivamente, las temáticas tratadas, los interlocutores implicados y el tipo de relación existente entre ellos, los contextos de referencia y las configuraciones lingüísticas seleccionadas para transmitir los significados textuales son todas variables que contribuyen activamente a la construcción del discurso (Chierichetti/Garofalo 2013: 12).

Son precisamente las pautas fraseológicas metafóricas el eje vertebrador de la presente investigación, cuyo aspecto innovador reside no tanto en las tipologías de fraseologismos estudiados sino en la metodología empleada (véase §4). Las (pocas) investigaciones existentes sobre fraseología metafórica en el lenguaje del turismo (como por ejemplo Navarro Coy/Soto Almela 2014), por un lado confirman la centralidad atribuida a fraseologismos en la comunicación turística, por el otro señalan que dichas unidades no están marcadas como pertenecientes a los géneros discursivos turísticos porque se encuentran en la lengua general. Expresiones idiomáticas como 'hacérsele a alguien la boca agua', 'ser un as en la manga', 'estar para comérselo', 'chuparse los dedos', etc. (para una descripción detallada, véanse 2014: 140142) no se encuentran con exclusividad en el lenguaje sectorial turístico, dado que forman parte del acervo fraseológico de la lengua general. Esto quiere decir que es difícil estudiar dichas unidades como parte de las redes discursivas que se crean en el texto turístico, debido a que carecen de connotación especializada.

En el presente estudio se analizan locuciones y colocaciones metafóricas (véase $\S 4$ ), para comprobar cómo las metáforas contribuyen a la caracterización de las entidades espaciales en los textos turísticos y si estas unidades fraseológicas forman parte del acervo especial del discurso turístico o de la lengua general.

La relación entre fraseología y metáfora es fundamental. Si, por un lado, la fraseología estructura el espacio del conocimiento y sus relaciones conceptuales, actuando a nivel textual, 
las metáforas intervienen a nivel conceptual, sustentando dichas relaciones y dibujando el escenario ${ }^{2}$, o sea el contexto en el que se inscribe el evento comunicativo.

Por lo que se refiere al nivel conceptual, la célebre definición de metáfora de Lakoff/Johnson "understanding and experiencing one kind of thing in terms of another" (1980:5) ha desplazado el interés de investigación de las expresiones lingüísticas metafóricas a la construcción conceptual de las metáforas.

De hecho, para la Teoría de la Metáfora Conceptual (TMC), el objeto de análisis no son las palabras utilizadas para dar vida a las expresiones metafóricas (o los medios lingüísticos explotados por ellas), sino más bien la naturaleza de la interacción entre los dominios conceptuales implicados en las correspondencias. Según la teoría lakoffiana, expresiones metafóricas como 'sus posiciones son indefendibles' o 'derribó todos mis argumentos' son pruebas de un mapa conceptual subyacente entre el dominio conceptual de la discusión (dominio meta) y el dominio de la guerra (dominio origen); la existencia de este mapa conceptual hace que podamos pensar y hablar acerca de las discusiones 'como si fueran batallas físicas'.

Pues bien, mediante la presente investigación empírica se ha intentado comprobar cómo las metáforas conceptuales basadas en UUFF quedan (meta)representadas en los textos para caracterizar los referentes espaciales y describir mundos imaginarios (Mattiello 2012: 71) en la mente del turista

\section{Datos}

Para el análisis se ha seleccionado un subcorpus del corpus Linguaturismo, elaborado en el ámbito del proyecto "Il linguaggio della comunicazione turistica spagnolo-italiano. Aspetti lessicali, pragmatici e interculturali" (Calvi 2011). Se trata de un corpus bilingüe españolitaliano representativo de los géneros discursivos de la comunicación turística tanto en su vertiente más divulgativa (con textos dirigidos al viajero, como guías, folletos, páginas web, etc., o escritos por los viajeros mismos, como foros y blog de viajeros), como en sus formas más especializadas relacionadas con la gestión del turismo (como contratos, informes, normativas, etc.).

De acuerdo con los objetivos de este estudio, para el análisis se ha seleccionado un subcorpus de textos en español dirigidos al viajero, que consta de los macrogéneros indicados en la Tabla $1^{3}$.

\begin{tabular}{|l|l|}
\hline Macrogénero & N. tokens \\
\hline Guías de turismo publicadas por editoriales & 129.121 \\
\hline Reportajes publicados en revistas de turismo & 70.678 \\
\hline Blog de viajes & 11.090 \\
\hline
\end{tabular}

\footnotetext{
2 "El término escenario se suele utilizar en los estudios discursivos para referirse, a través de esa metáfora teatral o cinematográfica, a los elementos físicos en los que se produce un determinado evento comunicativo, es decir, básicamente, el espacio y el tiempo y su organización. Estos elementos [...] forman una parte fundamental, aunque no la única, de lo que se denomina el contexto" (Calsamiglia/Tusón 2012: 91).

${ }^{3}$ Para una descripción de la taxonomía de los textos incluidos en el corpus, véanse Calvi (2011) y Mapelli/Piccioni (2011).
} 


\begin{tabular}{|l|l|}
\hline Foros de viajeros & 11.249 \\
\hline Folletos publicados por instituciones & 424.501 \\
\hline $\begin{array}{l}\text { Anuncios publicados en revista por institu- } \\
\text { ciones }\end{array}$ & 1.828 \\
\hline Anuncios publicados en web institucionales & 1.514 \\
\hline $\begin{array}{l}\text { Guías descriptivas publicadas en web institu- } \\
\text { cionales }\end{array}$ & 82.727 \\
\hline TOT. & $\mathbf{7 3 2 . 7 0 8}$ \\
\hline
\end{tabular}

Tabla 1: Subcorpus del corpus Linguaturismo utilizado para el análisis

En su conjunto el subcorpus consta de 732.708 tokens, anotados morfosintácticamente y lematizados con el programa FreeLing (Carreras et al. 2004). Para el análisis se ha utilizado el programa de interrogación AntConc (Anthony 2014).

\section{$4 \quad$ Método}

Desde el punto de vista metodológico, se ha adoptado un enfoque basado en corpus ${ }^{4}$ o, mejor dicho y citando las palabras de Hunston, un 'enfoque fraseológico' (2006: 55), ya que se ha explorado el corpus para posteriormente formular una hipótesis basada en las secuencias recurrentes observadas.

Para empezar, utilizando AntConc (Anthony 2014), se han seleccionado los N-gramas (es decir, conjuntos de palabras que pueden aparecer juntas en un texto, con un orden consecutivo determinado, Navarro Colorado 2015: 22) de una longitud de entre 3 y 6 palabras con una frecuencia superior o igual a 10.

El paso siguiente ha sido seleccionar los $\mathrm{N}$-gramas potencialmente metafóricos, utilizando el procedimiento propuesto por el Grupo Pragglejaz (MIP, Metaphor Identification Procedure, Pragglejaz Group 2007). Según este procedimiento, una unidad léxica es metafórica si se utiliza con un sentido que contrasta con un sentido existente más 'básico' de la misma palabra/expresión. En línea con los principios de la Teoría de la Metáfora Conceptual, un significado es 'más básico' si es “more concrete (what they evoke is easier to imagine, see, hear, feel, smell, and taste); related to bodily action; more precise (as opposed to vague); historically older" (Pragglejaz Group 2007: 3). Un ejemplo es la locución preposicional 'a través de', que se usa en combinación con sustantivos que indican espacio (a través de la pared, de la frontera, etc.) y sustantivos que indican tiempo (a través de los siglos, la historia, etc.): el sentido espacial se puede considerar más ‘básico’ por ser más concreto (más fácil de percibir a través de los sentidos) y más preciso. El sentido temporal sería, por lo tanto, metafórico.

Como muestran este ejemplo y otros presentados en el análisis, el procedimiento utilizado resulta ser un criterio 'conservativo', que identifica como metafóricos casos de metáforas catacresizadas o muertas, en los que el sentido metafórico puede ya estar recogido como un

\footnotetext{
${ }^{4}$ Se comparte la postura de McEnery/Xiao/Tono (2006: 11) que proponen el término corpus-based como denominador común para ambos enfoques (corpus-driven y corpus-based), frente a otros enfoques basados exclusivamente en la intuición (intuition-based), donde no se hace uso del corpus (Corpas Pastor 2008: 54-55).
} 
sentido más de la palabra (p. ej., capital [de la sidra]). En nuestro análisis se consideran metafóricos a partir de los criterios semánticos e históricos establecidos en la MIP.

Tras identificar los $\mathrm{N}$-gramas potencialmente metafóricos, se han clasificado en base al tipo de unidad fraseológica que conforman. A este respecto y por lo que atañe a la dimensión fraseológica del estudio, a raíz de la falta de unanimidad sobre qué es una unidad fraseológica y de las distintas clasificaciones que se encuentran en literatura, el estudio sienta sus bases en una acepción de fraseología más amplia, que se inspira más a los postulados de la lingüística de corpus que a las clasificaciones lexicográficas tradicionales (véase Pontrandolfo 2013: 6786), en línea con el enfoque 'fraseológico' preconizado por Hunston (2006). En este sentido, se comparte la definición de unidad fraseológica (UF) proporcionada por Gries que define un 'fraseologismo' como la co-occurrencia de una forma o de un lema de un elemento léxico y uno o más de un elemento lingüístico adicional de distintos tipos que funciona como una unidad semántica en una frase u oración y cuya frecuencia de co-ocurrencia es mayor que la que se esperaría por casualidad" (2008: 6) [traducción nuestra]. Para las finalidades de la investigación llevada a cabo, se ha integrado esta definición con la tripartición de UUFF propuesta por Corpas Pastor (1996) (véase Figura 1).

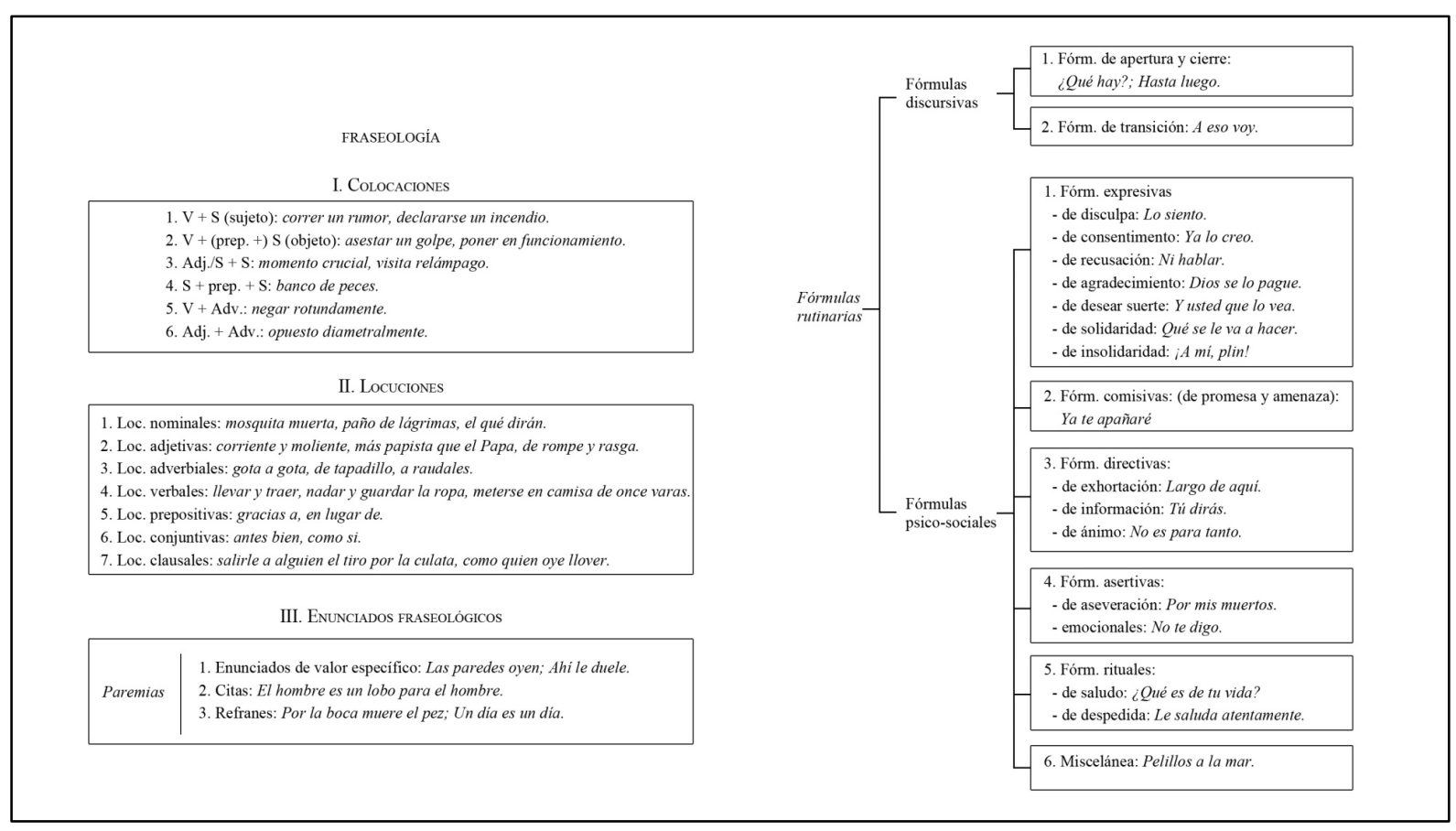

Figura 1: Las tres esferas fraseológicas según Corpas Pastor (1996: 270-271)

Cabe señalar que el análisis se ha centrado en las colocaciones y locuciones, al considerar como ya se ha adelantado en $§ 2$ - que son las UUFF que más contribuyen a urdir el tejido metafórico de los textos turísticos analizados en el corpus.

La Tabla 2 ejemplifica el listado de $\mathrm{N}$-gramas detectados antes mediante la función $\mathrm{N}$-grams del programa AntConc y luego clasificados según la tripartición de Corpas Pastor (1996) (véase Figura 1).

\begin{tabular}{|l|l|l|l|}
\hline Posición & Frecuencia & N-grama & Tipo de UF \\
\hline 14 & 231 & a lo largo & locución preposicional \\
\hline 25 & 182 & a través de & locución preposicional \\
\hline
\end{tabular}




\begin{tabular}{|c|c|c|c|}
\hline 67 & 113 & junto a la & locución preposicional \\
\hline 68 & 112 & en torno a & locución preposicional \\
\hline 76 & 105 & a partir de & locución preposicional \\
\hline 144 & 74 & merece la pena & locución verbal \\
\hline 182 & 65 & al aire libre & locución adverbial \\
\hline 256 & 53 & que cuenta con & $\varnothing$ \\
\hline 267 & 51 & en forma de & locución adverbial \\
\hline 268 & 51 & frente a la & locución preposicional \\
\hline 276 & 50 & cerca de la & locución preposicional \\
\hline 342 & 45 & a los pies & locución preposicional \\
\hline 360 & 44 & a la entrada & locución preposicional \\
\hline 399 & 41 & al pie de & locución adverbial \\
\hline 440 & 39 & a la altura & locución preposicional \\
\hline 442 & 39 & a orillas del & locución adverbial \\
\hline 465 & 38 & a base de & locución preposicional \\
\hline 497 & 37 & de entrada a & locución adverbial \\
\hline 509 & 37 & se alza el & colocación SUST+V \\
\hline 514 & 36 & a lo largo de los & locución preposicional \\
\hline 522 & 36 & en busca de & locución preposicional \\
\hline 529 & 36 & la desembocadura del & locución nominal \\
\hline 605 & 34 & que se extiende & colocación SUST+V \\
\hline 646 & 32 & el corazón de & locución nominal \\
\hline 688 & 31 & imagen de la & $\varnothing$ \\
\hline 694 & 31 & la salida de & $\varnothing$ \\
\hline 707 & 30 & a finales de & locución adverbial \\
\hline 708 & 30 & a su vez & locución adverbial \\
\hline 709 & 30 & alto de la & $\varnothing$ \\
\hline 797 & 29 & mayor parte de & $\varnothing$ \\
\hline 838 & 28 & la capital del & $\varnothing$ \\
\hline 842 & 28 & [a lo] largo de [los siglos] & locución preposicional \\
\hline 852 & 28 & punto de encuentro & locución nominal \\
\hline 863 & 27 & al lado de & locución preposicional \\
\hline 883 & 27 & en el centro de la & locución preposicional \\
\hline 898 & 27 & nos encontramos con & colocación V+SUST \\
\hline 900 & 27 & otro lado de & $\varnothing$ \\
\hline 902 & 27 & por encima de & locución preposicional \\
\hline 910 & 26 & a mediados del & locución preposicional \\
\hline 911 & 26 & a modo de & locución preposicional \\
\hline 921 & 26 & Casa de las & $\varnothing$ \\
\hline 1065 & 25 & el casco urbano & locución nominal \\
\hline 1066 & 25 & [en] el Centro de & locución preposicional \\
\hline 1091 & 25 & llama la atención & locución verbal \\
\hline
\end{tabular}


De 3-gramas a 6-gramas (frecuencias de corte: 10)

Número total de types: 5470

Número total de tokens: 118968

Tabla 2: Ejemplo de N-gramas extraídos del corpus Linguaturismo y clasificados fraseológicamente

En cuanto a la dimensión metafórica, el primer paso del análisis ha consistido en recuperar, a partir de las concordancias de cada $\mathrm{N}$-grama, los sintagmas enteros en su plena realización léxica en el corpus (identificando, por ejemplo, todos los casos en que a lo largo de se utiliza en combinación con sustantivos que indican TIEMPO en el corpus: a lo largo de los siglos, a lo largo de la Edad Media, a lo largo de aquel agitado siglo XIX italiano, etc.).

Dada la importancia conceptual del espacio en los textos turísticos, se ha decidido restringir el análisis a las expresiones metafóricas relacionadas con este dominio conceptual y sus subdominios (ENTIDADES GEOGRÁFICAS, URBANAS, ARQUITECTÓNICAS, etc.).

A cada expresión metafórica se ha asignado una metáfora conceptual formulada, según la tradición lakoffiana, a través de un paradigma del tipo EL DOMINIO META ES EL DOMINIO ORIGEN (por ejemplo, EL TIEMPO ES DINERO). Paralelamente, atendiendo a la formulación lingüística de las metáforas y siguiendo la terminología propuesta por Richards (1936), se considera que cada expresión consta de un tenor (no necesariamente expresado) correspondiente al objeto/fenómeno del que se habla y un vehículo metafórico, perteneciente al dominio origen y correspondiente a otro objeto/fenómeno cuyos atributos o rasgos se atribuyen al tenor.

A continuación, en $\S 5$ se ilustran las principales metáforas conceptuales identificadas y sus correspondientes expresiones lingüísticas; sucesivamente, en $\S 6$ se propone un análisis conceptual de las expresiones metafóricas, para investigar los procesos cognitivos a ellas asociados y sus efectos en el lector de textos turísticos.

\section{$5 \quad$ Metáforas de entidades espaciales}

El dominio conceptual del espacio y demás elementos espaciales (ENTIDAD GEOGRÁFICA o ENTIDAD URBANA) contribuyen a la creación de expresiones metafóricas como dominio meta (es decir, como espacio físico representado metafóricamente) y como dominio origen (es decir, como vehículo metafórico utilizado para representar metafóricamente elementos conceptuales no espaciales). Una tercera categoría de usos metafóricos corresponde a expresiones en las cuales la entidad espacial es contemporáneamente vehículo (dominio origen) y tenor (dominio meta) de la metáfora. En lo que sigue, se ilustran las tres categorías de usos por separado.

\subsection{El espacio como dominio meta}

Las representaciones metafóricas del espacio se realizan principalmente a través de personificaciones. La mayoría de estas utiliza metáforas corporales (EL ESPACIO ES CUERPO [HUMANO]), en las que las distintas entidades geográficas/espaciales se presentan como entidades dotadas de un cuerpo tendencialmente antropomorfo. Las UUFF a través de las cuales se expresan estas metáforas corporales tienden a ser locuciones preposicionales utilizadas principalmente para localizar los referentes espaciales, como en los ejemplos que siguen:

(1) $[\ldots]$ a los pies de dos pequeñas montañas

(2) $[\ldots]$ al pie de la espectacular funivía

ISSN 1615-3014 
(3) Varios restaurantes a pie de playa

En otros casos las metáforas antropomorfas se realizan a través de colocaciones que identifican una parte del referente espacial:

(4) el centro neurálgico de la ciudad

(5) un verdadero pulmón verde en la anatomía urbana de Madrid

(6) el pulso de la ciudad se halla en la Plaza de la Signoria

(7) la vida corre por las arterias urbanas de la calle Real

(8) El cinturón verde

(9) La Plaza es sin duda el corazón de la ciudad.

(10) [... ] la puerta del brazo meridional del crucero conserva toda la rica iconografía románica de la época gloriosa de Compostela.

(11) [...] en el mismo casco de las poblaciones

(12) la boca de la ría de Viveiro

Otro recurso utilizado para la creación de personificaciones son los verbos de movimiento ficticio ('fictive motion verbs', Talmy 2000) utilizados en colocaciones SUSTANTIVO [ENTIDAD ESPACIAL] + VERBO [MOVIMIENTO] en sustitución de expresiones existenciales, como en los siguientes ejemplos:

(13) [...] la llanura manchega se alza en La Mesa de Ocaña [...]

(14) Un excelente y amplio golfo se extiende ante Cagliari [...]

(15) Junto a él se levanta el emblemático teatro Arriaga [...]

(16) Parte una senda / una senda que asciende / atraviesa / baja / bordea / continúa / desciende / discurre / pasa / recorre / se interna / va / etc.

(17) calle adoquinada que sale a la derecha

(18) El camino gira a la izquierda

(19) A dos pasos, se abre la PLAZA DE LAS TENDILLAS

(20) el cerro sobre el que se asienta la villa

El movimiento antropomorfizado está también a la base de una serie de locuciones preposicionales:

(21) el Po en su camino pausado hacia el Adriático

(22) el rio Júcar a su paso por Alcalá del Júcar

Las personificaciones que sustituyen expresiones existenciales se realizan también a través de colocaciones en las cuales un sustantivo que indica entidad espacial/geográfica se combina con verbos que indican [DAR] o [CONTENER]. En el primer caso la experiencia turística se presenta como un regalo ofrecido por el destino turístico al viajero beneficiario de la donación, mientras que, en el segundo caso, el verbo subraya el valor o la preciosidad del objeto custodiado:

(23) Cataluña ofrece al visitante una gran variedad de productos culturales

(24) esta ciudad nos brinda una excepcional gastronomía

(25) el Palacio Episcopal, que alberga el Museo Diocesano

(26) La Biblioteca Estense de Módena conserva la Biblia de Borso d'Este 
Las personificaciones en otros casos se sirven de locuciones nominales y colocaciones SUSTANTIVO [ENTIDAD ESPACIAL] + VERBO [NACER] para indicar el punto de origen de la entidad espacial aludida:

(27) El carril / la carretera / la trama urbana / el río, etc. nace

(28) el nacimiento del río Cuervo

Por último, hay una serie de verbos que es complicado encasillar en una categoría semántica única: al utilizarse con entidades espaciales, dan vida a personificaciones que apuntan a las funciones vitales del ser humano:

(29) una ciudad que se nutre de románticas leyendas

\subsection{El espacio como dominio origen}

A esta categoría de usos se pueden asociar las expresiones que identifican el espacio y el desplazamiento en el espacio como vehículos metafóricos. En otras palabras, se trata de expresiones en las cuales la experiencia turística o uno de sus aspectos se convierten en viaje metafórico.

Un primer ejemplo de este tipo lo ofrece el paradigma metafórico EL TIEMPO ES ESPACIO, que se realiza a través de locuciones preposicionales y nominales:

(30) a lo largo de toda su historia

(31) muchos pueblos la han visitado a través de la historia

(32) un atractivo recorrido por la historia del medievo a través de sus tres salas

(33) el visitante no sólo realiza un espectacular viaje en el tiempo [...]

(34) este breve paseo por la historia

Este paradigma metafórico da pie a una serie de extensiones metafóricas, como EL TRANSCURRIR DEL TIEMPO ES DESPLAZAMIENTO EN EL ESPACIO o LO ANTIGUO ES LEJANO, que se realizan lingüísticamente a través de locuciones nominales y verbales como en los siguientes ejemplos:

(35) también resisten el paso del tiempo

(36) la entrada de la primavera

(37) que también viajan en el tiempo sensual de la Florencia dormida

(38) regresando en el tiempo

(39) han llegado intactos hasta nuestros días

(40) Nos permiten acercarnos a la historia

(41) Este itinerario permite que el turista viva la ciudad desde un punto de vista remoto en el tiempo

Destacan también una serie de locuciones nominales, en las que el espacio es utilizado como vehículo metafórico para la VIDA (MATERIAL O ESPIRITUAL) y los ARTEFACTOS que son productos identificativos de un lugar:

(42) Centro de la vida cultural bilbaína

(43) este centro de saber y conocimiento

(44) centro de la fe católica

(45) capital de la sidra

(46) Es la capital de un queso sin parangón 


\subsection{El espacio como dominio origen y dominio meta}

En esta categoría de usos se incluyen dos tipos principales de expresiones metafóricas: por un lado, aquellas expresiones en las que tanto el dominio origen como el dominio meta son entidades espaciales, aunque de distinto tipo/dimensión; por otro lado, las expresiones en las que el vehículo metafórico admite también una interpretación literal.

El primer tipo de expresiones se concreta sobre todo en el paradigma metafórico LAS ENTIDADES GEOGRÁFICAS SON EDIFICIOS, que se realiza a través de locuciones preposicionales y nominales como en los siguientes ejemplos:

(47) a la entrada de Bilbao

(48) ya estaba a las Puertas de Nápoles

(49) La calle Morería Baja, en las Puertas de Murcia, antigua entrada a la ciudad medieval, guarda los restos de una columna romana

(50) Bielsa, puerta de entrada al maravilloso valle de Fineta.

(51) Beteta es, además, la puerta del Parque Natural del Alto Tajo

(52) que discurre por toda la cornisa cantábrica

(53) Las Islas Canarias han sido un puente de enlace entre Europa.

El segundo tipo lo ejemplifican locuciones preposicionales y nominales y colocaciones en las cuales las entidades espaciales pueden tener una doble interpretación literal y metafórica:

(54) Cruce de caminos durante toda su historia

(55) Ruta del aceite

(56) [...] ruta del románico

(57) [...] se encuentra un santuario de la gastronomía del Véneto

(58) [...] la frontera con el mundo del islam

(59) [...] vivir en la frontera

(60) A la sombra de la Catedral Oviedo

(61) a la sombra de su impresionante monasterio

Estos casos se consideran al mismo tiempo literales y metafóricos porque la clara intención metafórica de los usos se acompaña a su plausibilidad literal. Por ejemplo, en el caso de a la sombra de, el hecho de que esta locución preposicional indique metafóricamente proximidad espacial no descarta la posibilidad de que algo esté literalmente a la sombra (p. ej. de una catedral); es distinto el caso de a los pies de una catedral, donde la realidad ontológica excluye una interpretación literal de la expresión.

\subsection{El espacio como dominio origen y dominio meta}

A nivel textual, aunque la metodología utilizada no permite observar la distribución e interacción de las expresiones metafóricas en los textos, la frecuencia (>10) de los n-gramas analizados evidencia algunos de los esquemas de distribución textual de las metáforas descritos en literatura (p. ej., Semino 2008; Dorst 2017). Entre estos destacan:

- Repetición de los vehículos metafóricos (Semino 2008: 23, p. ej., a lo largo, arteria, pulmón, etc.); 
- Recurrencia ("use of different expressions relating to the same broad source domain" 2008: 23): este mecanismo permite la exploración del dominio origen a través de sus elementos constitutivos (p. ej., el dominio origen del cuerpo que resulta muy productivo: pulmón, arteria, pie, pulso, corazón, brazo, casco, boca, centro neurálgico, cinturón, anatomía urbana, etc.);

- Extensión ("when at least two metaphorically used words belonging to different phrases describe the same target domain/scenario in terms of the same source domain/scenario" 2008: 25): este mecanismo es evidente en el caso del paradigma metafórico EL TIEMPO ES ESPACIO con sus extensiones metafóricas EL TRANSCURRIR DEL TIEMPO ES DESPLAZAMIENTO EN EL ESPACIO o LO ANTIGUO ES LEJANO: p. ej., llegados intactos hasta nuestros días, regresando en el tiempo, remoto en el tiempo;

- Interacción entre sentido literal y metafórico de una expresión (Semino 2008): se trata de un fenómeno observado con frecuencia en textos literarios y definido también como 'literalización de los vehículos' ("literalisation of vehicles", Goatly 2011: 290-298) o 'realización metafórica' (Hrushovski 1984); consiste en la coexistencia de dos planos referenciales (el literal y el metafórico), que contribuye a aumentar la polivalencia de la expresión metafórica. En nuestro corpus lo hemos observado en casos como vivir en la frontera, la ruta del vino, donde las metáforas no son incompatibles con una interpretación literal, ya que esta es ontológicamente posible.

\subsection{El espacio como dominio origen y dominio meta}

El análisis de las UUFF asociadas con el concepto de espacio en un corpus de comunicación turística ha permitido arrojar nueva luz sobre las maneras en que este macro-ámbito conceptual es utilizado en textos turísticos como dominio meta y dominio origen. En el primer caso, destacan sobre todo UUFF que realizan metáforas antropomorfas a través de personificaciones, como por ejemplo la metáfora del cuerpo (las arterias urbanas, a pie de playa, etc.) y metáforas basadas en el uso de verbos de movimiento ficticio (una senda que discurre, el camino gira a la izquierda, etc.). Mediante estas expresiones el espacio geográfico se representa a través de conceptos concretos, delimitados, tangibles $\mathrm{y}$, por tanto, fácilmente accesibles al universo cognitivo del lector/viajero. En el segundo caso, en cambio, el espacio se convierte en vehículo metafórico para representar de forma concreta conceptos más abstractos, como puede ser el tiempo (a través de los siglos, viajan en el tiempo, etc.), convirtiendo la experiencia turística en un viaje metafórico.

La selección de $N$-gramas con frecuencia mínima de 10 como punto de partida del análisis ha permitido identificar algunos patrones recurrentes de uso. A través de estos, el dominio origen adquiere relevancia cognitiva y se asocia automáticamente al dominio meta. Esto hace que las ciudades se conceptualicen naturalmente como cuerpos humanos con varias partes funcionales y que resulte difícil hablar del tiempo sin referirse al espacio u otros dominios concretos (Lakoff/Johnson, 1980). La frecuencia de metáforas de este tipo genera la que Darian (2000: 173) denomina 'reciprocidad' (reciprocity), es decir la automatización de las correspondencias metafóricas más frecuentes. En el caso de los textos turísticos, la creación y/o explotación de asociaciones conceptuales 'automáticas' favorecen la fácil fruición del texto turístico, convirtiendo el destino turístico en una entidad más accesible desde el punto de vista cognitivo. Además, estos mecanismos otorgan visibilidad a los dominios origen y, a través de ellos, a los 
elementos concretos que los integran: estos, por su naturaleza, tienen una mayor prominencia cognitiva y contribuyen a crear un mayor impacto sensorial con "el efecto de sumir al turista en un ambiente cargado de múltiples sugestiones sensoriales” (Calvi 2011: 211).

La prominencia cognitiva del espacio y sus metáforas se hace aún más patente en las colocaciones en las que las entidades espaciales se asocian con verbos de movimiento ficticio (p. ej., la llanura manchega se alza, Un excelente y amplio golfo se extiende, etc.). Se trata de expresiones que representan un estado ([- dinámico] [- control]) como si fuera un proceso ([+ dinámico] [- control]) (Dik 1997, en Jiménez Martínez-Losa 2006). De hecho, los sujetos de estos verbos no cambian, ni se mueven, pero se describen como si lo estuvieran haciendo, llevando al lector/viajero a percibirlo así: en otras palabras, "[m]otion does not actually take place but is subjectively construed in the interpretation of the reader" (Cappelli 2012: 25).

A nivel expresivo, el uso de estos verbos y de las correspondientes personificaciones otorga dinamismo a los referentes, al mismo tiempo que contribuye a destacar alguna propiedad normalmente positiva - del objeto representado (p. ej., un castillo que se alza se representa implícitamente como majestuoso, Piccioni/Biscu 2009). Desde la perspectiva cognitiva, los verbos de movimiento ficticio guían la mirada del lector, que imagina y simula mentalmente el movimiento (Matlock 2004, en Cappelli 2012); de esta forma, el sujeto que percibe la imagen se coloca en el centro del escenario cognitivo, que es explorado egocéntricamente a partir de una perspectiva subjetiva (Wallentin et al. 2005, en Cappelli 2012). Como señala Cappelli (2012: 32): "The reader-experiencer is ideally brought to the centre of the scene: the imaginary movement happens with respect to his line of sight". El resultado es una mayor implicación del lector en lo dicho, con expresiones que, al proporcionar información espacial, también propician la percepción sensorial y emocional del referente.

\section{Conclusiones}

La investigación que se ha llevado a cabo ha demostrado la importancia de locuciones y colocaciones figuradas, hasta ahora poco estudiadas en la comunicación turística, confirmando el papel fundamental que la metáfora desempeña en el discurso turístico, en línea con los resultados de otras investigaciones empíricas (entre otros, Francesconi 2008; Manca 2008). Además, el trabajo ha confirmado la utilidad metodológica y la eficacia de los N-gramas (véase §) a la hora de detectar patrones fraseológicos potencialmente metafóricos, así como la relevancia de los corpus para la identificación de las metáforas (véase Semino 2017). Se trata de una metodología que bien se podría aplicar a otros géneros discursivos.

Por lo que se refiere a las UUFF metafóricas efectivamente utilizadas en el corpus, se confirman los resultados de Navarro Coy/Soto Alela (2014), ya que la mayoría de los fraseologismos detectados no son exclusivos de la comunicación turística porque se encuentran también en la lengua general. En efecto, se trata de metáforas convencionales cuyo uso no se puede considerar prototípico del discurso turístico.

Desde el punto de vista conceptual, se ha comprobado que las metáforas del espacio tienen una doble función: por un lado, familiarizar al turista con el espacio evocando esquemas cognitivos conocidos y cognitivamente accesibles, poniendo la experiencia del espacio al alcance del lector/viajero; por el otro, utilizar la prominencia cognitiva del espacio como elemento concreto 
para describir la experiencia turística, guiando la mirada del lector/viajero y convirtiéndolo en sujeto activo de la experiencia. Todo esto contribuye a poner al lector en el centro de la experiencia sensorial generando efectos persuasivos, ya señalados como centrales en los textos turísticos (véase, p. ej., Suau 2011).

El trabajo propuesto no tiene pretensión alguna de exhaustividad. Al contrario, se presenta como una base para profundizar aún más en el tema desde otras perspectivas. Sería interesante, por ejemplo, desglosar los resultados obtenidos por géneros discursivos, para comprobar si la frecuencia de las UUFF varía según las tipologías de productos turísticos y los géneros discursivos. Se puede suponer, por ejemplo, que la función del uso metafórico cambia entre los textos turísticos editoriales de corte más tradicional y los textos turísticos redactados por los usuarios (turismo 2.0, como blogs y foros): más cognitivo y experiencial en el primer caso, más pragmático en el segundo. Otra dimensión de análisis podría fundarse en un estudio de corte contrastivo (p. ej. español vs. italiano, español vs. inglés), para verificar si las dinámicas textuales varían entre culturas diferentes (véase, a este respecto, el estudio de corte contrastivo de Manca 2017).

Lo que no va a cambiar es, sin duda alguna, la función persuasiva de las UUFF metafóricas basadas en el espacio, uno de los elementos que más contribuye a generar la imagen del destino turístico en la mente del turista y a invitarlo a visitar esos espacios. El estudio ha demostrado que dichas unidades se basan en un componente icónico empleado para (meta)representar un lugar, una atmósfera, un estado de ánimo, con el objetivo final de conseguir efectos suasorios.

\section{Referencias bibliográficas}

Anthony, Lawrence (2014): AntConc (Version 3.4.3) [Computer Software]. Tokyo, Waseda University. http://www.laurenceanthony.net/ [09/11/2017].

Barcelona Sánchez, Antonio/Rocamora Abellán, Rafael (2000): "El argot turístico y la teoría cognitiva de la metáfora y la metonimia”. Cuadernos de Turismo, N. 5/2000, 19-34. http://revistas.um.es/turismo/article/view/22811/22101_[09/11/2017].

Calsamiglia, Helena/Tusón, Amparo (2012): Las cosas del decir. Manual de análisis del discurso. $3^{\text {a }}$ edición. Barcelona: Ariel Letras.

Calvi, Maria Vittoria (2000): Il linguaggio spagnolo del turismo. Viareggio: Mario Baroni Editore.

Calvi, Maria Vittoria (2006): Lengua y comunicación en el español del turismo. Madrid: Arco/Libros S. L.

Calvi, Maria Vittoria (2009): "El lenguaje del turismo" En: Calvi, Maria Vittoria et al. (eds.): Las lenguas de especialidad en español. Roma, Carocci: 199-224.

Calvi, Maria Vittoria (2011): "Pautas de análisis para los géneros del turismo". En: Calvi, Maria Vittoria/Mapelli, Giovanna (eds.): La lengua del turismo. Géneros discursivos y terminología. Bern, Lang: 19-45.

Cappelli, Gloria (2012): “Travelling in Space: Spatial Representation in English and Italian Tourism Discourse”. Textus Vol. XXV, N. 1 (January-April). Roma, Carocci: 19-35.

Carreras, Xavier/Chao, Isaac/Padró, Lluís/Padró, Muntsa (2004): "Freeling: An open-source suite of language analyzers". Proceedings of the 4th International Conference on Language Resources and Evaluation (LREC '04), 26th-28th May 2004, Lisbon/Paris, ELRA: 239-242. 
Caruso, Valeria (2016): "Lavoro che fai, lingua che usi. La dimensione figurativa del lessico finanziario inglese”. En: Dal Maso, Elena/Navarro, Carmen (eds.): Gutta cavat lapidem. Indagini fraseologiche e paremiologiche. Mantova, Universitas Studiorum: 49-512.

Chierichetti, Luisa/Garofalo, Giovanni (2013): "Introducción”. En Chierichetti, Luisa/Garofalo, Giovanni (eds.): Discurso profesional y lingüística de corpus. Perspectivas de investigación. Bergamo, Cerlis Series: CELSB, 9-25.

Corbacho Sánchez, Alfonso (2014): "Die metaphorische Konzeptualisierung der Wirtschaft in touristischen Texten: Deutsch und Spanisch im Kontrast”. Muttersprache 124/1: 26-40.

Corpas Pastor, Gloria (1996): Manual de fraseología española. Madrid: Gredos.

Corpas Pastor, Gloria (2008): Investigar con corpus en traducción: los retos de un nuevo paradigma. Bern: Lang.

Dann, Graham (1996): The Language of Tourism. A Sociolinguistic Perspective, Wallingford: CAB International.

Darian, Steven (2000): “The role of figurative language in introductory science texts". International Journal of Applied Linguistics, 10(2), 163-186.

Dik, Simon C. (ed.) (1997): The theory of functional grammar. Part 1: The structure of the clause. Berlin/New York: Mouton de Gruyter.

Dobrovol'skij, Dmitrij (1998): "On Cultural Component in the Semantic Structure of Idioms". En: Durčo, Peter (ed.) (1998): Phraseology and Paremiology. Europhras 97: International Symposium. September 2-5 1997. Bratislava, Liptovsky: 55-61.

Dorst, Aletta (2017): “Textual Patterning of Metaphor". En: Semino, Elena/Demjén, Zsófia (eds.): The Routledge handbook of metaphor and language, Abingdon/New York, Routledge: 178-192.

Francesconi, Sabrina (2007): English for Tourism Promotion. Italy in British Tourism Texts. Milano: Hoepli.

Francesconi, Sabrina (2008): "Metaphors of Jewels as Strategies of Persuasion in British Tourist Promotional Texts". En: Garzone, Giuliana/Catenaccio, Paola (eds.): Language and Bias in Specialised Discourse. Milano, CUEM: 176-186.

Goatly, Andrew (2011): The Language of Metaphors. New York: Routledge.

Gries, Stefan Th. (2008): "Phraseology and Linguistic Theory: a brief survey". En: Granger, Sylviane/Meunier, Fanny (eds.): Phraseology: An Interdisciplinary Perspective. Amsterdam/Philadelphia, Benjamins: 3-25.

Hrushovski, Benhamin (1984): "Poetic Metaphor and Frames of Reference". Poetics today 5, 5-43.

Hunston, Susan (2006): "Phraseology and System: a Contribution to the Debate". En: Thompson, Paul/Hunston, Susan (eds.): System and Corpus. Exploring Connections, London \& Oakvielle: Equinox, 55-80.

Jiménez Martínez-Losa, Noelia (2006): “Towards a typology of fictive motion events: review of existing proposals and presentation of new perspectives". Interlingüística 17, 562-569.

Lakoff, George/Johnson, Mark (1980): Metaphors we live by. Chicago: University of Chicago Press.

Luque Durán, Juan de Dios/Manjón, Francisco José (1998): “Tipología léxica y tipología fraseológica: universales y particulares, metáfora y lenguaje taurino”. En: Luque Durán, Juan 
de Dios/Pamies Betrán, Antonio (eds.): Léxico y fraseología, Granada: Método Ediciones, 139-154.

Manca, Elena (2008): "From Phraseology to Culture: Qualifying Adjectives in the Language of Tourism". International Journal of Corpus Linguistics 13 (3): 368-385.

Manca, Elena (2017): "Persuasione, Tourist Gaze e turismo di lusso. Modi comunicativi e culture a confronto". Lingue Linguaggi 20: 215-237.

Mapelli, Giovanna/Piccioni, Sara (2011): “Taxonomía de los textos turísticos: Factores lingüísticos y factores contextuales". En: Calvi, Maria Vittoria / Mapelli, Giovanna (eds) La lengua del turismo. Géneros discursivos y terminología. Bern, Lang: 47-73.

McEnery, Tony/Xiao, Richiard/Tono, Yuko (eds.): (2006): Corpus-based Language Studies. Edinburgh: Edinburgh University Press.

Mattiello, Elisa (2012): "Metaphor in Tourism Discourse: Imagined Worlds in English Tourist Texts on the Web". Textus 25/1: 69-84.

Matlock, Teenie (2004): "Fictive Motion as Cognitive Simulation". Memory \& Cognition 32: 1389-1400.

Messina Fajardo, Luisa (2017): Apuntes de fraseología, paremiología, traducción y didáctica del español. Madrid: Avant.

Navarro Colorado, Borja (2015): Guía rápida de análisis de corpus (con AntConc). Universidad de Alicante. http://www.dlsi.ua.es/ borja/riilua/grac.pdf_[09/11/2017].

Navarro Coy, Marta/Soto Almela, Jorge (2014): "La traducción de la idiomaticidad en el contexto turístico español-inglés" Paremia 23: 135-146. http://cvc.cervantes.es/lengua/paremia/pdf/023/013_navarro-soto.pdf_[09/11/2017].

Pamies Bertrán, Antonio (2002): "Modelos icónicos y archimetáforas: algunos problemas metalingüísticos en el ámbito de la fraseología" Language Design 4: 9-20. http://elies.rediris.es/Language_Design/LD4/pamies.pdf [09/11/2017].

Piccioni, Sara/Biscu, Maria Giovanna (2009): "No te puedes perder... Estrategias de implicación del lector en un corpus de folletos turísticos españoles”. En: Cantos Gómez, Pascual/ Sánchez Pérez, Aquilino (eds.): A survey on corpus-based research - Un panorama de investigaciones basadas en corpus. Asociación Española de Lingüística de Corpus, 109-126.

Pontrandolfo, Gianluca (2013): La fraseología en las sentencias penales: un estudio contrastivo español, italiano, inglés basado en corpus. Tesis de doctorado no publicada. Universidad de Trieste. https://www.openstarts.units.it/handle/10077/8590 [14/12/2018].

Pragglejaz Group (2007): "MIP: A method for identifying metaphorically used words in discourse". Metaphor \& Symbol 22/1: 1-39.

Ramos-Ruiz, Ismael (2015): "El cáncer de la economía: La fraseología de las metáforas médicas periodísticas". Opción, Año 31, n. Especial 6 (2015), 721-741. http://www.redalyc.org/html/310/31045571044/ [09/11/2017].

Richards, Ivor Armstrong (1936): The Philosophy of Rhetoric. London: Oxford University Press.

Semino, Elena (2008): Metaphor in Discourse, Cambridge/New York: Cambridge University Press.

Semino, Elena (2017): “Corpus linguistics and metaphor”. En: Dancygier, Barbara (ed.): The Cambridge Handbook of Cognitive Linguistics. Cambridge, Cambridge University Press: 463-76. 
Suau Jiménez, Francisca (2011): "La persuasión a través del metadiscurso interpersonal en el género Página Web Institucional de Promoción Turística en inglés y español”. En: Calvi, Maria Vittoria/Mapelli, Giovanna (eds.): La lengua del turismo: géneros discursivos y terminología. Bern, Lang: 177-200.

Swales, John (1990): Genre Analysis: English in Academic and Research Settings. Cambridge: Cambridge University Press.

Talmy, Leonard (2000): Towards a Cognitive Semantics. 2 vols. Cambridge (MA): MIT Press. Urry, John (1990): The Tourist Gaze. Leisure and Travel in Contemporary Societies. London: Sage.

Urry, John (2001): Globalizing the Tourist Gaze, Department of Sociology, Lancaster University.

http://www.lancaster.ac.uk/fass/resources/sociology-online-papers/papers/urry-globalisingthe-tourist-gaze.pdf [09/11/2017].

Wallentin, Mikkel/Lund, Torben/Ostergaard, Svend/Ostergaard, Leif/Roepstorff, Andreas. (2005): "Motion Verb Sentences Activate Left Posterior Middle Temporal Cortex Despite Static Context". Neuroreport 16/6: 649-652. 